\title{
Asymptotic Behaviour of the Castelnuovo-Mumford Regularity
}

\author{
Dedicated to F. Hirzebruch on the occasion of his 70th birthday \\ S. DALE CUTKOSKY ${ }^{1 \star}$, JÜRGEN HERZOG ${ }^{2}$ and NGÔ VIÊT TRUNG ${ }^{3}$ \\ ${ }^{1}$ Department of Mathematics, University of Missouri, Columbia, MO 65211, U.S.A. \\ ${ }^{2}$ Fachbereich Mathematik, Universität-GHS Essen, Postfach 103764, Germany; \\ e-mail:mat300@uni-essen.de \\ ${ }^{3}$ Institute of Mathematics, Box 631, Bo Ho, Hanoi, Vietnam
}

(Received: 24 December 1997; accepted in final form: 21 April 1998)

\begin{abstract}
In this paper the asymptotic behaviour of the Castelnuovo-Mumford regularity of powers of a homogeneous ideal $I$ is studied. It is shown that there is a linear bound for the regularity of the powers $I^{n}$ whose slope is the maximum degree of a homogeneous generator of $I$, and that the regularity of $I^{n}$ is a linear function for large $n$. Similar results hold for the integral closures of the powers of $I$. On the other hand we give examples of ideals for which the regularity of the saturated powers is asymptotically not a linear function, not even a linear function with periodic coefficients.
\end{abstract}

Mathematics Subject Classifications (1991): 13C99, 13D45, 13 F17.

Key words: Regularity, powers of ideals, saturated powers, Rees algebra.

\section{Introduction}

Let $A=k\left[X_{1}, \ldots, X_{r}\right]$ be a polynomial ring over an arbitrary field $k$. Let $L$ be any finitely generated graded $A$-module. The Castelnuovo-Mumford regularity $\operatorname{reg}(L)$ of $L$ is defined to be the maximum degree $n$ for which there is an index $j$ such that $H_{\mathfrak{m}}^{j}(L)_{n-j} \neq 0$, where $H_{\mathfrak{m}}^{j}(L)$ denotes the $j$ th local cohomology module of $L$ with respect to the maximal graded ideal $\mathfrak{m}$ of $A$. It is also the maximum degree $n$ for which there is an index $j$ such that $\operatorname{Tor}_{j}^{A}(k, L)_{n+j} \neq 0$. The Castenuovo-Mumford regularity is an important invariant which measures the complexity of the given module. For instance, if

$$
0 \rightarrow \cdots \rightarrow F_{j} \rightarrow \cdots \rightarrow F_{1} \rightarrow F_{0} \rightarrow L \rightarrow 0
$$

is the minimal free resolution of $L$ over $A$ and if $a_{j}$ is the maximum degree of the generators of $F_{j}$, then

$$
\operatorname{reg}(L)=\max \left\{a_{j}-j \mid j \geqslant 0\right\} .
$$

\footnotetext{
$\star$ The first author was partially supported by NSF.
} 
See, e.g., Eisenbud and Goto [EG], Bayer and Mumford [BM] for more information on this notion.

Let $I$ be any homogeneous ideal of $A$. Recently, Swanson [S] has proved that there is a number $D$ such that for all $n \geqslant 1, \operatorname{reg}\left(I^{n}\right) \leqslant n D$. This result follows from a linear bound on the growth of associated primes of ideals which is closely linked with a version of the uniform Artin-Rees lemma along the line of Huneke's uniform bounds in noetherian rings [Hu2]. However, Swanson could not provide a formula for the number $D$ in general.

A possible candidate for $D$ is $\operatorname{reg}(I)$. In fact, if $\operatorname{dim} A / I=1$, Geramita, Gimigliano and Pittelloud [GGP] and Chandler [C] showed that $\operatorname{reg}\left(I^{n}\right) \leqslant n \operatorname{reg}(I)$ for all $n \geqslant 1$. This result can be easily generalized to the case $\operatorname{depth} A / I^{n} \geqslant$ $\operatorname{dim} A / I-1$ for all $n$. The same bound also holds for a Borel-fixed monomial ideal $I$ by the Eliahou-Kervaire resolution [EK]. See [SS] and [HT] for explicit linear bounds for $\operatorname{reg}\left(I^{n}\right)$ when $I$ is an arbitrary monomial ideal.

The problem of bounding $\operatorname{reg}\left(I^{n}\right)$ is also of interest in algebraic geometry. Given a projective variety $X \subset \mathbb{P}^{r}$, and let $\ell_{X}$ be the ideal sheaf of the embedding of $X$. The Castelnuovo-Mumford regularity of $\ell_{X}$ is defined to be the the least integer $t$ such that $H^{i}\left(\mathbb{P}^{r}, \ell_{X}(t-i)\right)=0$ for all $i \geqslant 1$. Let $d_{X}$ denote the minimum of the degrees $d$ such that $X$ is a scheme-theoretic intersection of hypersurfaces of degree at most $d$. For a smooth complex projective variety, Bertram, Ein and Lazarsfeld [BEL] have shown that there is a number $e$ such that $H^{i}\left(\mathbb{P}^{s}, l_{X}^{n}(a)\right)=0$, for all $a \geqslant n d_{X}+e, i \geqslant 1$. The proof used the Kodaira vanishing theorem. See [B] and $[\mathrm{W}]$ for related recent results.

In this paper we will propose a simpler method to estimate $\operatorname{reg}\left(I^{n}\right)$. The main result is the following.

THEOREM 1.1. Let I be an arbitrary homogeneous ideal. Let $d(I)$ denote the maximum degree of the homogeneous generators of I. Then

(i) There is a number e such that $\operatorname{reg}\left(I^{n}\right) \leqslant n d(I)+e$ for all $n \geqslant 1$.

(ii) $\operatorname{reg}\left(I^{n}\right)$ is a linear function for all $n$ large enough.

We can estimate the number $e$ (Theorem 2.4) and, if $I$ is generated by forms of the same degree, the place $n$ where $\operatorname{reg}\left(I^{n}\right)$ starts to be a linear function (Proposition 3.7).

We will also show that $d\left(I^{n}\right)$ is a linear function for $n \gg 0$. Since we always have $d(I) \leqslant \operatorname{reg}(I)$, it follows that

$$
\lim \frac{\operatorname{reg}\left(I^{n}\right)}{n}=\lim \frac{d\left(I^{n}\right)}{n} .
$$

It is clear that the common limit is a positive number $\leqslant d(I)$. Therefore, the difference between $\operatorname{reg}\left(I^{n}\right)$ and $n \operatorname{reg}(I)$ can be arbitrarily large if $d(I)<\operatorname{reg}(I)$.

Part (i) of the above result implies that for an arbitrary projective variety $X \subset$ $\mathbb{P}^{r}$, there is a number $e$ such that $H^{i}\left(\mathbb{P}^{s}, \ell_{X}^{n}(a)\right)=0$, for all $a \geqslant n d_{X}+e, i \geqslant 1$. 
However, part (ii) does not have a similar geometric version. In fact, it does not hold if we replace $I^{n}$ by its saturation $\widetilde{I^{n}}$, though $I^{n}$ and $\widetilde{I^{n}}$ define the same projective scheme. We will give examples of homogeneous ideals of 'fat' points for which reg $\left(\widetilde{I^{n}}\right)$ is not a linear function for large $n$ (Example 4.2). In particular, using a counter-example to Zariski's Riemann-Roch problem in positive characteristic [CS] we can construct an example such that $\operatorname{reg}\left(\widetilde{I}^{n}\right)$ is not even a linear polynomial with periodic coefficients (Example 4.3).

We also give an example of a homogeneous ideal in the coordinate ring of an abelian surface such that $\lim \operatorname{reg}\left(\widetilde{I^{n}}\right) / n$ is an irrational number (Example 4.4).

Our method is based on a natural bigrading of the Rees algebra $R=\oplus_{n \geqslant 0} I^{n} t^{n}$ given by setting $\operatorname{deg} x t^{n}=(\operatorname{deg} x, n)$ for all homogeneous element $x$ of $I^{n}$. It is not hard to see that

$$
H_{\mathfrak{m}}^{j}\left(I^{n}\right)_{a} \simeq H_{M}^{j}(R)_{(a, n)}, \quad \operatorname{Tor}_{j}^{A}\left(k, I^{n}\right)_{a} \simeq \operatorname{Tor}_{j}^{S}(S / N, R)_{(a, n)},
$$

for all numbers $a, n$, where $S=k\left[X_{1}, \ldots, X_{r}, Y_{1}, \ldots, Y_{s}\right]$ is the polynomial ring mapping onto $R$ with $Y_{i} \mapsto f_{i} t$ when $I$ is generated by the homogenous elements $f_{1}, \ldots, f_{s}$, and where $N=\left(Y_{1}, \ldots, Y_{s}\right)$. Therefore, we only need to study the bigraded structure of $H_{M}^{j}(R)$ and $\operatorname{Tor}_{j}^{S}(S / M, S)$ in order to estimate reg $\left(I^{n}\right)$.

The proof of Theorem 1.1(i) and (ii) will be found in Section 2 and Section 3, respectively. We would like to mention that (i) has been also obtained by LavilaVidal and Zarzuela by a different method (private communication) and that linear programming is used to prove (ii). The same method can also be applied to give linear bounds for reg $\left(\overline{I^{n}}\right)$, where $\overline{I^{n}}$ denotes the integral closure of $I^{n}$, and for $\operatorname{reg}\left(I_{1}^{n_{1}} \ldots I_{m}^{n_{m}}\right)$, where $I_{1}, \ldots, I_{m}$ are arbitrary homogeneous ideals. Moreover it can be shown that if the graded algebra $\bigoplus_{n \geqslant 0} \widetilde{I^{n}} t^{n}$ is finitely generated, then there are a finite number of linear functions such that $\operatorname{reg}\left(\tilde{I}^{n}\right)$ varies among these functions for $n \gg 0$ (Theorem 4.3).

\section{Linear Bound for the Regularity}

We begin with some observation on the bigraded structure of local cohomology modules which we shall need in the proof of Theorem 1.1(i).

Let $R=\oplus_{a, n \geqslant 0} R_{(a, n)}$ be a noetherian bigraded ring and $E=\oplus_{a, n \in \mathbb{Z}} E_{(m, n)}$ be a bigraded $R$-module. We may consider $R$ as an $\mathbb{N}$-graded ring with $R_{n}=$ $\oplus_{a \geqslant 0} R_{(a, n)}$ and $E$ as a $\mathbb{Z}$-graded module with $E_{n}=\oplus_{a \geqslant 0} E_{(a, n)}$. It is clear that $R_{0}$ is also an $\mathbb{N}$-graded ring and that $E_{n}$ is a graded $R_{0}$-module.

Let $\mathfrak{m}$ be the maximal graded ideal of $R_{0}$. Then the local cohomology module $H_{\mathfrak{m}}^{i}\left(E_{n}\right)$ is a well-defined graded $R_{0}$-module for all $i \geqslant 0$.

Let $M$ denote the ideal generated by the elements of $\mathfrak{m}$, i.e. $M=\oplus_{n \geqslant 0} \mathfrak{m} R_{n}$. We shall see that $H_{\mathfrak{m}}^{i}\left(E_{n}\right)$ is a $\mathbb{Z}$-graded component of the local cohomology module $H_{M}^{i}(E)$.

LEMMA 2.1. $H_{\mathfrak{m}}^{i}\left(E_{n}\right)_{a}=H_{M}^{i}(E)_{(a, n)}$ for all numbers $a, n$. 
Proof. We shall use the characterization of local cohomology modules by means of the Koszul complexes (see e.g. [BH], [H1]). Let $x_{1}, \ldots, x_{r}$ be a family of generating elements for $\mathfrak{m}$. Set $\mathbf{x}^{t}=x_{1}^{t}, \ldots, x_{r}^{t}$ and denote by $H^{i}\left(\mathbf{x}^{t}, \cdot\right)$ the $i$ th cohomology of the Koszul complex functor associated with $\mathbf{x}^{t}$. Then

$$
H_{\mathfrak{m}}^{i}\left(E_{n}\right)=\lim _{\longrightarrow} H^{i}\left(\mathbf{x}^{t}, E_{n}\right), \quad H_{M}^{i}(E)=\underline{\lim } H^{i}\left(\mathbf{x}^{t}, E\right) .
$$

Since the elements $x_{1}, \ldots, x_{r}$ have degree zero in the $\mathbb{N}$-graded ring $R$, we have $H^{i}\left(\mathbf{x}^{t}, E_{n}\right)=H^{i}\left(\mathbf{x}^{t}, E\right)_{n}$. From this it follows that $H_{\mathfrak{m}}^{i}\left(E_{n}\right)=\lim _{\longrightarrow} H^{i}\left(\mathbf{x}^{t}, E\right)_{n}=$ $H_{M}^{i}(E)_{n}$. It is clear that the equation $H_{\mathfrak{m}}^{i}\left(E_{n}\right) \simeq H_{M}^{i}(E)_{n}$ also reflects the bigraded structure in the sense that $H_{\mathfrak{m}}^{i}\left(E_{n}\right)_{a}=H_{M}^{i}(E)_{(a, n)}$.

From now on let $R=\oplus_{n \geqslant 0} I^{n} t^{n}$ be the Rees algebra of a homogeneous ideal $I$ in a polynomial ring $A=k\left[X_{1}, \ldots, X_{r}\right]$. As $I$ is homogeneous, we may view $R$ as a bigraded ring with $R_{(a, n)}=\left(I^{n}\right)_{a} t^{n}$.

Let $\mathfrak{m}=\left(X_{1}, \ldots, X_{r}\right)$ be the maximal graded ideal of $A$. By Lemma 2.1 we have $H_{\mathfrak{m}}^{i}\left(I^{n}\right)_{a}=H_{M}^{i}(R)_{(a, n)}$, for all numbers $a, n$. Therefore, we may get information on the graded structure of $H_{\mathfrak{m}}^{i}\left(I^{n}\right)$ by the bigraded structure of $H_{M}^{i}(R)$.

Assume that $I$ is generated by $s$ homogeneous polynomials. Then $R$ may be represented as a factor ring of the bigraded polynomial ring $S=k\left[X_{1}, \ldots, X_{r}, Y_{1}, \ldots\right.$, $Y_{s}$ ]. Let $N$ denote the ideal of $S$ generated by $X_{1}, \ldots, X_{r}$. It is clear that

$$
H_{M}^{i}(R)_{(a, n)} \simeq H_{N}^{i}(R)_{(a . n)},
$$

for all numbers $a, n$. We will use a bigraded minimal free resolution of $R$ over $S$ to study the the bigraded structure of $H_{N}^{i}(R)$.

First we have the following description of $H_{N}^{i}(S)$.

\section{LEMMA 2.2.}

$$
\begin{aligned}
& H_{N}^{i}(S)=0, \quad i \neq r, \\
& H_{N}^{r}(S)=k\left[X_{1}^{\alpha_{1}} \ldots X_{r}^{\alpha_{r}} Y_{1}^{\beta_{1}} \ldots Y_{s}^{\beta_{s}} \mid \alpha_{1}, \ldots, \alpha_{r}<0 ; \beta_{1}, \ldots, \beta_{s} \in \mathbb{N}\right] .
\end{aligned}
$$

Proof. Since $S$ is a direct product of copies of $A=k\left[X_{1}, \ldots, X_{r}\right]$, we have $H_{N}^{i}(S)=H_{\mathfrak{m}}^{i}(A) \otimes_{A} S$. It is well-known [H1] that

$$
H_{\mathfrak{n}}^{i}(A)=0, \quad i \neq r, \quad H_{\mathfrak{n}}^{r}(A)=k\left[X_{1}^{\alpha_{1}} \ldots X_{r}^{\alpha_{r}} \mid \alpha_{1}, \ldots, \alpha_{r}<0\right] .
$$

Hence the conclusion is immediate.

Let $d_{1}, \ldots, d_{s}$ be the degree of the homogeneous generators of $I$. Then the bigrading of the polynomial ring $S$ is given by

bideg $X_{i}=(1,0), \quad i=1, \ldots, r$, 
$\operatorname{bideg} Y_{j}=\left(d_{j}, 1\right), \quad j=1, \ldots, s$.

This can be used to obtain information on the bigraded vanishing of $H_{N}^{r}(S)$.

COROLLARY 2.3. $H_{N}^{r}(S)_{(a, n)}=0$ for all $a \geqslant n d(I)-r+1$.

Proof. Note that $d(I)=\max \left\{d_{1}, \ldots, d_{s}\right\}$. Since

$$
\begin{aligned}
& \operatorname{bideg} X_{1}^{\alpha_{1}} \ldots X_{r}^{\alpha_{r}} Y_{1}^{\beta_{1}} \ldots Y_{s}^{\beta_{s}} \\
& \quad=\left(\alpha_{1}+\cdots+\alpha_{r}+\beta_{1} d_{1}+\cdots+\beta_{s} d_{s}, \beta_{1}+\cdots+\beta_{s}\right),
\end{aligned}
$$

using Lemma 2.2 we get

$$
\begin{aligned}
H_{N}^{r}(S)_{(a, n)}= & k\left[X_{1}^{\alpha_{1}} \ldots X_{r}^{\alpha_{r}} Y_{1}^{\beta_{1}} \ldots Y_{s}^{\beta_{s}} \mid \alpha_{1}, \ldots, \alpha_{r}<0 ;\right. \\
& \left.\alpha_{1}+\cdots+\alpha_{r}+\beta_{1} d_{1}+\cdots+\beta_{s} d_{s}=a, \beta_{1}+\cdots+\beta_{s}=n\right] .
\end{aligned}
$$

If $a \geqslant n d(I)-r+1$, then

$$
\begin{aligned}
\alpha_{1}+\cdots+\alpha_{r} & =a-\left(\beta_{1} d_{1}+\cdots+\beta_{s} d_{s}\right) \\
& \geqslant a-\left(\beta_{1}+\cdots+\beta_{s}\right) d(I)=a-n d(I) \geqslant 1-r .
\end{aligned}
$$

Hence at least one of the numbers $\alpha_{1}, \ldots, \alpha_{r}$ must be nonnegative. From this it follows that $H_{N}(S)_{(a, n)}=0$.

The following result gives Theorem 1.1 (i) by setting $E=R$. This result will be used to give a linear bound for reg $\left(\overline{I^{n}}\right)$, too.

THEOREM 2.4. Let E be an arbitrary finitely generated bigraded module over the Rees algebra of I. Let

$$
\begin{aligned}
0 & \rightarrow \cdots \rightarrow \oplus_{t} S\left(-a_{t j},-b_{t j}\right) \rightarrow \cdots \rightarrow \oplus_{t} S\left(-a_{t 1},-b_{t 1}\right) \\
& \rightarrow \oplus_{t} S\left(-a_{t 0},-b_{t 0}\right) \rightarrow E \rightarrow 0
\end{aligned}
$$

be a bigraded minimal free resolution of $E$ over $S$, where $S$ is defined as above. Put $c_{j}=\max _{t}\left\{a_{t j}-b_{t j} d(I)\right\}$ and $e=\max \left\{c_{j}-j \mid j=0, \ldots, r\right\}$. For all $n \geqslant 1$ we have $\operatorname{reg}\left(E_{n}\right) \leqslant n d(I)+e$.

Proof. First we will study the graded vanishing of $H_{N}^{i}(E), i=1, \ldots, r$. Rewrite the above resolution of $E$ as follows

$$
0 \rightarrow \cdots \rightarrow F_{j} \rightarrow \cdots \rightarrow F_{1} \rightarrow F_{0} \rightarrow E \rightarrow 0 .
$$

Let $K_{j}$ denote the image of the map $F_{j} \rightarrow F_{j-1}$ for $j \geqslant 1$. Then there are the exact sequences

$$
0 \rightarrow K_{j} \rightarrow F_{j-1} \rightarrow K_{j-1} \rightarrow 0,
$$


where $K_{0}=E$. Consider the derived exact sequence of local cohomology modules of these exact sequences. For $i<r$, we use Lemma 2.2 to deduce that

$$
H_{N}^{i}(E) \simeq H_{N}^{i+1}\left(K_{1}\right) \simeq \cdots \simeq H_{N}^{r-1}\left(K_{r-i-1}\right)
$$

and that there is an injective map $H_{N}^{r-1}\left(K_{r-i-1}\right) \rightarrow H_{N}^{r}\left(K_{r-i}\right)$ and a surjective map $H_{N}^{r}\left(F_{r-i}\right) \rightarrow H_{N}^{r}\left(K_{r-i}\right)$. For $i=r$ we also have a surjective map $H_{N}^{r}\left(F_{0}\right) \rightarrow$ $H_{N}^{r}(E)$. Therefore, for all $i \geqslant 0, H_{N}^{i}(E)_{(m, n)}=0$ if $H_{N}\left(F^{r-i}\right)_{(m, n)}=0$. By Corollary 3.2, $H_{N}^{r}\left(S\left(-a_{t j},-b_{t j}\right)\right)_{(m, n)}=0$, for $m-a_{t j} \geqslant\left(n-b_{t j}\right) d-r+1$, where $d=d(I)$. Therefore, $H_{N}^{r}\left(F^{r-i}\right)_{(m, n)}=0$ if $m \geqslant\left(n-b_{t r-i}\right) d+a_{t r-i}-r+1$ for all $t$. The latter condition is satisfied if $m \geqslant n d+c_{r-i}-r+1$. Hence $H_{N}^{i}(E)_{(m, n)}=0$, for all $m \geqslant n d+c_{r-i}-r+1$.

By Lemma 2.1 we get $H_{\mathfrak{m}}^{i}\left(E_{n}\right)_{m-i}=H_{N}(E)_{(m-i, n)}=0$, for $m-i \geqslant n d+$ $c_{r-i}-r+1, i=1, \ldots, r$. Since $n d+e \geqslant n d+c_{r-i}-r+i$, this vanishing holds if $m \geqslant n d+e$. Note that $H_{\mathfrak{m}}^{0}\left(E_{n}\right)=0$. Then we obtain $\operatorname{reg}\left(E_{n}\right) \leqslant n d+e$.

COROLLARY 2.5. Let $X \subset \mathbb{P}^{r}$ be an arbitrary projective variety. Let $\ell_{X}$ be the ideal sheaf of the embedding and $d_{X}$ the minimum of the degrees $d$ such that $X$ is a scheme-theoretic intersection of hypersurfaces of degree at most $d$. Then there is a number e such that $H^{i}\left(\mathbb{P}^{r}, \ell_{X}^{n}(a)\right)=0$, for all $a \geqslant n d_{X}+e, i \geqslant 1$.

Proof. Let $I$ be a homogeneous ideal generated by forms of degree at most $d_{X}$ such that $\ell_{X}$ is the ideal sheaf associated with $I$. Then $d(I)=d_{X}$. By Theorem 1.1 (i) there is an integer $e$ such that $H_{\mathfrak{m}}^{i}\left(I^{n}\right)_{a}=0$ for $a \geqslant n d_{X}+e, i \geqslant 0$. Therefore the conclusion.

COROLLARY 2.6. Let I be a homogeneous ideal generated by s elements. Assume that the Rees algebra of I is Cohen-Macaulay. Then

$$
\left.\operatorname{reg}\left(I^{n}\right) \leqslant n d(I)+(s-1)[d(I)-1)\right],
$$

for all $n \geqslant 1$.

Proof. The assertion follows immediately from the bound $a_{t j} \leqslant s d(I)-(s-$ 1) $+j, j \geqslant 0$, for $E=R$ given by O. Lavila-Vidal [L, Proposition 4.1], in case the Rees algebra of $I$ is Cohen-Macaulay.

There are several important classes of ideals for which one knows that their Rees algebras are Cohen-Macaulay, see e.g. Eisenbud and Huneke [EH].

EXAMPLE 2.7. Let $I$ be the ideal generated by the maximal minors of a generic $p \times q$ matrix, $p \leqslant q$. Then the Rees algebra of $I$ is a Cohen-Macaulay ring [EH]. Therefore

$$
\operatorname{reg}\left(I^{n}\right) \leqslant n p+\left[\left(\begin{array}{l}
q \\
p
\end{array}\right)-1\right](p-1),
$$


for all $n \geqslant 1$. This is far from being the actual value of $\operatorname{reg}\left(I^{n}\right)$. Akin, Buchsbaum, and Weyman $[\mathrm{ABW}]$ already gave a linear resolution for $I^{n}$ from which it follows that $\operatorname{reg}\left(I^{n}\right)=n p$ for all $n \geqslant 1$. We are grateful to A. Conca for this information.

If we set $E=\oplus_{n \geqslant 0} \overline{I^{n}} t^{n}$, where $\overline{I^{n}}$ denotes the integral closure of $I^{n}$, then $E$ is a finitely generated bigraded $R$-module with $E_{n} \simeq \overline{I^{n}}$. Hence from Theorem 2.4 we also obtain a linear bound for $\operatorname{reg}\left(\overline{I^{n}}\right)$.

PROPOSITION 2.8. Let I be an arbitrary homogeneous ideal. Then there is a number e such that $\operatorname{reg}\left(\overline{I^{n}}\right) \leqslant n d(I)+e$ for all $n \geqslant 1$.

\section{Asymptotic Behaviour of Regularity}

Let $I$ be a homogeneous ideal in $A=k\left[X_{1}, \ldots, X_{r}\right]$. In this section we will show that $\operatorname{reg}\left(I^{n}\right)$ is not only bounded by a linear function, but, for $n \gg 0$, is a linear function. The approach will be similar as in the previous section.

For any $A$-module $L$ we set $\operatorname{reg}_{i}(L)=\max \left\{a \mid \operatorname{Tor}_{i}(k, L)_{a} \neq 0\right\}-i$. Since $\operatorname{reg}(L)=\max \left\{\operatorname{reg}_{i}(L) \mid i \geqslant 0\right\}$, Theorem 1.1 (ii) follows from the next result.

THEOREM 3.1. Let I be an arbitrary homogeneous ideal. Then for all $i \geqslant 0$, the function $\operatorname{reg}_{i}\left(I^{n}\right)$ is linear for $n \gg 0$.

Recall that for any homogeneous ideal $J, d(J)$ denotes the maximal degree of the homogeneous generators of $J$. It is well-known that $d(J)$ is nothing else than $\operatorname{reg}_{0}(J)$. The next result encodes the fact that the linear functions associated with $\operatorname{reg}_{0}\left(I^{n}\right)$ and $\operatorname{reg}\left(I^{n}\right)$ have the same slope.

COROLLARY 3.2. Let I be an arbitrary homogeneous ideal. Then

$$
\lim \frac{d\left(I^{n}\right)}{n}=\lim \frac{\operatorname{reg}\left(I^{n}\right)}{n},
$$

and this common limit is a positive integer $\leqslant d(I)$.

Proof. Let reg $\left(I^{n}\right)=a n+b$ and $\operatorname{reg}_{0}\left(I^{n}\right)=c n+d$ for $n \gg 0$. Since $\operatorname{reg}_{0}\left(I^{n}\right) \leqslant$ $\operatorname{reg}\left(I^{n}\right)$ for all $n$, it follows that $c \leqslant a$. On the other hand, by Theorem 1.1 (i) we have $\operatorname{reg}\left(I^{m n}\right) \leqslant \operatorname{reg}_{0}\left(I^{n}\right) m+e$ for large $n$ and all $m \geqslant 0$. This implies that $a n \leqslant \operatorname{reg}_{0}\left(I^{n}\right)=c n+d$ for all large $n$. Therefore, $a \leqslant c$, and so $a=c$. It is clear that $c$ is a positive integer $\leqslant d(I)$.

In order to prove Theorem 3.1 we shall consider the Rees algebra $R=\oplus_{n \geqslant 0} I^{n} t^{n}$ as a factor ring of the bigraded polynomial ring $S=k\left[X_{1}, \ldots, X_{r}, Y_{1}, \ldots, Y_{s}\right]$ as in Section 3. Let $\mathfrak{m}=\left(X_{1}, \ldots, X_{r}\right)$ be the maximal graded ideal of $A$ and $N=\mathfrak{m} S$.

LEMMA 3.3. Let $E$ be a finitely generated bigraded $R$-module. Put $E_{n}=$ $\oplus_{a \in \mathbb{Z}} E_{(a, n)}$. Then $\operatorname{Tor}_{i}^{A}\left(k, E_{n}\right)_{a} \simeq \operatorname{Tor}_{i}^{S}(S / N, E)_{(a, n)}$, for all $a, n$ and $i \geqslant 0$. 
Proof. Consider a graded minimal free $S$-resolution of the $R$-module $E$

$$
\mathbb{F}: \quad 0 \rightarrow \cdots \rightarrow F_{j} \rightarrow \cdots \rightarrow F_{1} \rightarrow F_{0} \rightarrow E \rightarrow 0 .
$$

Taking the $n$-homogeneous component is an exact functor, so that the sequence

$$
\mathbb{F}_{n}: \quad 0 \rightarrow \cdots \rightarrow\left(F_{j}\right)_{n} \rightarrow \cdots \rightarrow\left(F_{1}\right)_{n} \rightarrow\left(F_{0}\right)_{n} \rightarrow E_{n} \rightarrow 0
$$

is exact. Since the modules $\left(F_{i}\right)_{n}$ are free $A$-modules, $\mathbb{F}_{n}$ is a free $A$-resolution for $E_{n}$. We have $\operatorname{Tor}_{i}^{S}(S / N, E)=H_{i}(\mathbb{F} / \mathfrak{m} \mathbb{F})$ so that $\operatorname{Tor}_{i}^{S}(S / N, E)_{n} \simeq H_{i}\left(\mathbb{F}_{n} / \mathfrak{m} \mathbb{F}_{n}\right)$ which is isomorphic to $\operatorname{Tor}_{i}^{A}\left(k, E_{n}\right)$. Hence $\operatorname{Tor}_{i}^{A}\left(k, E_{n}\right)_{a} \simeq \operatorname{Tor}_{i}^{S}(S / N, E)_{(a, n)}$.

Remark. The above free resolution $\mathbb{F}_{n}$ of $E_{n}$ is not minimal in general. For instance, let $I=\left(X_{1}^{2}, X_{1} X_{2}, X_{2}^{2}\right) \subset A=k\left[X_{1}, X_{2}\right]$. Then $R=S /\left(f_{1}, f_{2}, f_{3}\right)$ with $f_{1}=X_{2} Y_{1}-X_{1} Y_{2}, f_{2}=X_{2} Y_{2}-X_{1} Y_{3}$ and $f_{3}=Y_{2}^{2}-Y_{1} Y_{3}$. One sees easily that $\left(f_{1}, f_{2}, f_{3}\right)$ is a height 2 perfect ideal, and hence the Rees algebra $R$ has the $S$-resolution

$$
0 \rightarrow S(-5,-2)^{2} \rightarrow S(-3,-1)^{2} \oplus S(-4,-2) \rightarrow S \rightarrow R \rightarrow 0 .
$$

Thus, if we want to compute a resolution of $I^{2}$, we have to take the second component of the above resolution, and get

$$
0 \rightarrow A(-5)^{2} \rightarrow A(-5)^{6} \oplus A(-4) \rightarrow A(-4)^{6} \rightarrow I^{2} \rightarrow 0,
$$

which, of course, is not minimal.

By Lemma 3.3 we have

$$
\operatorname{reg}_{i}\left(I^{n}\right)=\max \left\{a \mid \operatorname{Tor}_{i}^{S}(S / N, R)_{(a, n)} \neq 0\right\}-i .
$$

Notice that each $\operatorname{Tor}_{i}(S / N, R)$ is a finitely generated bigraded module over the bigraded polynomial ring $S / N=k\left[Y_{1}, \ldots, Y_{s}\right]$ with bideg $Y_{i}=\left(d_{i}, 1\right), i=1, \ldots, s$. Then Theorem 3.1 follows from the following property of such modules.

THEOREM 3.4. Let $E$ be any finitely generated bigraded module over $k\left[Y_{1}, \ldots, Y_{s}\right]$. The function $\rho_{E}(n):=\max \left\{a \mid E_{(a, n)} \neq 0\right\}$ is linear for $n \gg 0$.

Proof. Put $T=k\left[Y_{1}, \ldots, Y_{s}\right]$. It is clear that for a given exact sequence of bigraded $T$-modules $0 \rightarrow E^{\prime \prime} \rightarrow E \rightarrow E^{\prime} \rightarrow 0$, we have $\rho_{E}(n)=$ $\max \left\{\rho_{E^{\prime \prime}}(n), \rho_{E^{\prime}}(n)\right\}$ for all $n \in \mathbb{N}$. Therefore, since there exists a sequence of bigraded submodules

$$
0=E_{0} \subset E_{1} \subset \cdots \subset E_{i-1} \subset E_{i}=M
$$

of $E$ such that $E_{j} / E_{j-1}$ is cyclic for $j=1, \ldots, i$, we may assume that $E$ is cyclic. 
We represent $E$ as a quotient $T / J$. Let $<$ be any term order, and denote by in $(J)$ the initial ideal of $J$ with respect to this term order. It is clear that $T / J$ has a $k$ basis consisting of the residues classes of all the monomials which do not belong to in $(J)$, and it is well-known that the residue classes of the same monomials modulo $J$ form a (bigraded) $k$-basis of $T / J$. Therefore $\rho_{E}(n)=\rho_{T / \text { in }(J)}(n)$ for all $n \geqslant 0$, and we may assume that $J$ itself is a monomial ideal.

Let $J$ be generated by the monomials $Y_{1}^{c_{i 1}} \ldots Y_{s}^{c_{i s}}$ for $i=1, \ldots, p$. For any $\mathbf{a}=\left(a_{1}, \ldots, a_{s}\right) \in \mathbb{N}^{s}$ let $y^{\mathbf{a}}$ denote the residue class of $Y_{1}^{a_{1}} \ldots, Y_{s}^{a_{s}}$ in $T / J$. Let $B_{n}$ denote the minimal basis of $(T / J)_{n}$. Then $\rho_{E}(n)=\max \left\{v(\mathbf{a}) \mid y^{\mathbf{a}} \in B_{n}\right\}$, with $v(\mathbf{a})=\sum_{i} a_{i} d_{i}$. Note that $y^{\mathbf{a}} \in B_{n}$ if and only if $\sum_{j} a_{j}=n$, and for all $i=1, \ldots, p$ there exists an integer $1 \leqslant j \leqslant s$ with $a_{j}<c_{i j}$.

Let $L$ denote the set of maps $\{1, \ldots, p\} \rightarrow\{1, \ldots, s\}$, and consider for each $f \in L$ the subset

$$
B_{n, f}=\left\{y^{\mathbf{a}} \mid \sum_{j} a_{j}=n, a_{f(i)}<c_{i f(i)} \text { for } i=1, \ldots, s\right\} .
$$

It is clear that $B_{n}=\cup_{f \in L} B_{n, f}$. Define $\rho_{f}(\mathbf{a})=\max \left\{v(\mathbf{a}) \mid y^{\mathbf{a}} \in B_{n, f}\right\}$. Then $\rho_{E}(n)=\max \left\{\rho_{f}(n) \mid f \in L\right\}$. Thus it suffices to show that the functions $\rho_{f}(n)$ are linear for all $f \in F$ and all $n \gg 0$.

Let $\left\{j_{1}, \ldots, j_{k}\right\}$ be the image of $f$, and suppose that $j_{1}<j_{2} \cdots<j_{k}$. We set $c_{j_{t}}=\min \left\{c_{i j(i)} \mid j(i)=j_{t}\right\}-1$ for $t=1, \ldots, k$. Then

$$
B_{n, f}=\left\{y^{\mathbf{a}} \mid \sum_{j} a_{j}=n \text { and } a_{j_{t}} \leqslant c_{j_{1}}, \text { for } t=1, \ldots, k\right\} \text {, }
$$

and $\rho_{f}(n)$ is given by the maximum of the linear functional $v(\mathbf{a})$ on the convex bounded set

$$
C_{n}=\left\{\mathbf{a} \mid \sum_{j} a_{j}=n, \text { and } a_{j_{t}} \leqslant c_{j_{1}} \text { for } t=1, \ldots,\right\} .
$$

This is a rather trivial example of linear programming. The solution is the following.

Suppose that $\ell$ is the smallest integer such that $j_{t}=t$ for $t<\ell$ and $j_{\ell}>\ell$. In other words, we have $a_{1}<c_{1}, \ldots, a_{\ell-1}<c_{\ell-1}$ and no bound on $a_{\ell}$ (except that $\left.\sum_{j} a_{j}=n\right)$.

If $\ell=s+1$, then $\sum_{j} a_{j}$ can be at most $\sum_{j} c_{j}$, so that for $n \gg 0, B_{n, f}=0$ and hence $\rho_{f}(n)=0$.

If $\ell \leqslant s$, let $n \geqslant c_{1}+c_{2}+\ldots+c_{\ell-1}$. We claim that $v$ has its maximal value for $\mathbf{a}=\left(c_{1}, \ldots, c_{\ell-1}, n-\sum_{j=1}^{\ell-1} c_{j}, 0, \ldots, 0\right)$. Then

$$
v(\mathbf{a})=\sum_{j=1}^{\ell-1} d_{j} c_{j}+d_{\ell}\left(n-\sum_{j}^{\ell-1} c_{j}\right),
$$


which is a linear function on $n$, as we wanted to show.

Indeed, if $\mathbf{a}=\left(a_{1}, \ldots, a_{s}\right) \in C_{n}$, and if for some $1 \leqslant i<j \leqslant s$ we have $a_{i}<c_{i}$ and $a_{j}>0$, then $\mathbf{a}^{\prime}=\left(a_{1}, \ldots, a_{i}+1, \ldots, a_{j}-1, \ldots, a_{s}\right)$ also belongs to $C_{n}$ and $v\left(\mathbf{a}^{\prime}\right) \geqslant v(\mathbf{a})$ since $d_{i} \geqslant d_{j}$, by assumption. This argument shows that if we fill up the first 'boxes' as much as possible, we must reach the maximal value of $v$. The resulting a with maximal value is exactly the one described above.

Theorem 3.4 also has the following interesting consequence

COROLLARY 3.5. Let I be an arbitrary homogeneous ideal. Then $\operatorname{reg}\left(\overline{I^{n}}\right)$ is a linear function for $n \gg 0$.

Proof. Put $E=\oplus_{n \geqslant 0} \bar{I}^{n} t^{n}$. Then $E$ is a finitely generated bigraded module over the Rees algebra of $I$ with $E_{n} \simeq \overline{I^{n}}$. By Lemma 3.3 we have $\operatorname{reg}_{i}\left(E_{n}\right)=$ $\rho_{\operatorname{Tor}_{i}^{S}(S / N, E)}(n)$ for all $i \geqslant 0$. Since $\operatorname{reg}\left(E_{n}\right)=\max \left\{\operatorname{reg}_{i}\left(E_{n}\right) \mid i \geqslant 0\right\}$, the conclusion follows from Theorem 3.4.

Remark With the same method as above one can prove the prove the following modifications of Theorem 3.1: Let $I_{1}, \ldots, I_{m}$ be graded ideals in the polynomial ring $A$. Then there exist integers $a_{1}, \ldots, a_{m}$ with $a_{j} \leqslant d\left(I_{j}\right)$ for $j=1, \ldots, m$, and an integer $b$ such that $\operatorname{reg}\left(I_{1}^{n_{1}} \ldots I_{m}^{n_{m}}\right)=a_{1} n_{1}+\cdots+a_{m} n_{m}+b$, for all $n_{1}, \ldots, n_{m} \gg$ 0 . For the proof one considers the multi-Rees ring $A\left[I_{1} t_{1}, \ldots, I_{m} t_{m}\right]$.

Now we will estimate the place where $\operatorname{reg}\left(I^{n}\right)$ starts to be a linear function when $I$ is generated by forms of the same degree. We shall need the following observation.

LEMMA 3.6. Let $0 \rightarrow E \rightarrow F \rightarrow G \rightarrow 0$ be an exact sequence of graded A-modules.

(i) If $\operatorname{reg}(E)>\operatorname{reg}(G)+1$, then $\operatorname{reg}(F)=\operatorname{reg}(E)$.

(ii) If $\operatorname{reg}(E)<\operatorname{reg}(G)+1$, then $\operatorname{reg}(F)=\operatorname{reg}(G)$.

Proof. Consider the derived long exact sequence

$$
H_{\mathfrak{m}}^{i-1}(G) \rightarrow H_{\mathfrak{m}}^{i}(E) \rightarrow H_{\mathfrak{m}}^{i}(F) \rightarrow H_{\mathfrak{m}}^{i}(G) \rightarrow H_{\mathfrak{m}}^{i+1}(E)
$$

Put $n=\max \{\operatorname{reg}(E), \operatorname{reg}(G)\}$. It is obvious that $\operatorname{reg}(F) \leqslant n$.

If $\operatorname{reg}(E)>\operatorname{reg}(G)+1$, then $n=\operatorname{reg}(E)$. We choose $i$ such that $H_{\mathfrak{m}}^{i}(E)_{n-i} \neq 0$. Since $\operatorname{reg}(G)<n-1, H_{\mathfrak{m}}^{i-1}(G)_{n-i}=0$. Hence $H_{\mathfrak{m}}^{i}(F)_{n-i} \neq 0$. From this it follows that $\operatorname{reg}(F)=n$.

If $\operatorname{reg}(E)<\operatorname{reg}(G)+1$, then $n=\operatorname{reg}(G)$. We choose $i$ such that $H_{\mathfrak{m}}^{i}(G)_{n-i} \neq 0$. Since reg $(E) \leqslant n, H_{\mathfrak{m}}^{i+1}(E)_{n-i}=0$. Hence $H_{\mathfrak{m}}^{i}(F)_{n-i} \neq 0$.

Our estimation depends on the minimum number of generators of $I$ and the Castelnuovo-Mumford regularity $\operatorname{reg}(R)$ of the Rees algebra $R=\oplus_{n \geqslant 0} I^{n} t^{n}$ as a $\mathbb{N}$-graded ring with the usual grading $\operatorname{deg} x t^{n}=n, x \in I^{n}$. The regularity 
$\operatorname{reg}(R)$ can be computed in terms of certain minimal set of generators of $I$ [T]. For instance, if $I$ is generated by a $d$-sequence [Hu1], then $\operatorname{reg}(R)=0$.

Recall that the Castelnuovo-Mumford regularity $\operatorname{reg}(E)$ of a graded module $E$ over any $\mathbb{N}$-graded ring $B$ is defined to be the largest integer $n$ for which there exists an index $i$ such that $H_{B_{+}}^{i}(E)_{a-i} \neq 0$, where $B_{+}$is the ideal of $B$ generated by the homogeneous elements of positive degree.

If we consider $R$ as a $\mathbb{N}$-graded module over the $\mathbb{N}$-graded polynomial ring $S$ with $\operatorname{deg} X_{i}=0$ and $\operatorname{deg} Y_{j}=1$, then $\operatorname{reg}(R)=\max \left\{b_{t j}-j \mid j \geqslant 0\right\}$, where $b_{t j}$ are the second coordinates of the bidegree of the generators of the $j$ th term of a minimal bigraded free resolution of $R$ over $S$.

PROPOSITION 3.7. Let I be a homogeneous ideal generated by sorms of the same degree d. Put $c=\operatorname{reg}(R)+s+1$. Then, for $n \geqslant c, \operatorname{reg}\left(I^{n}\right)=(n-c) d+$ $\operatorname{reg}\left(I^{c}\right)$.

Proof. We need to modify the statement as follows. Let $S=k\left[X_{1}, \ldots, X_{n}\right.$, $\left.Y_{1}, \ldots, Y_{s}\right]$ be a bigraded polynomial ring with $\operatorname{bideg} X_{i}=(1,0)$ and $\operatorname{bideg} Y_{j}=$ $(d, 1)$, where $d>0$ is a fixed integer. For any finitely generated bigraded $S$-module $E$ let $E_{n}=\oplus_{a \in \mathbb{Z}} E_{(a, n)}$. Then $S$ is an $\mathbb{N}$-graded ring and $E$ an $\mathbb{Z}$-graded $S$-module. Put $c=\operatorname{reg}(E)+s+1$. We claim that for $n \geqslant c, \operatorname{reg}\left(E_{n}\right)=(n-c) d+\operatorname{reg}\left(E_{c}\right)$.

Since $R$ may be considered as a finitely generated bigraded $S$-module with $R_{n} \simeq$ $I^{n}$, the conclusion clearly follows from this claim.

If $s=0, S_{n}=0$ for all $n>0$. It follows that $\operatorname{reg}(E)=\max \left\{n \mid E_{n} \neq 0\right\}$. Hence $E_{n}=0$ for $n \geqslant \operatorname{reg}(E)+1$. In this case, $d=0$.

To prove the claim in the case $s>0$ we may assume that the base field $k$ is infinite. Then we can find a linear form $Y$ in $Y_{1}, \ldots, Y_{s}$ such that $Y \notin P$ for any associated prime $P \nsupseteq\left(Y_{1}, \ldots, Y_{s}\right)$ of $E$. In other words, $Y$ is a filter-regular element of $E$ with respect to the ideal $\left(Y_{1}, \ldots, Y_{s}\right)$. Note that $Y$ is a bihomogeneous form with $\operatorname{bideg} Y=(d, 1)$. Put $K=E / 0_{E}: Y$. Consider the exact sequence of graded $A$-modules:

$$
0 \rightarrow K_{n-1}(-d) \stackrel{Y}{\longrightarrow} E_{n} \rightarrow[E / Y E]_{n} \rightarrow 0 .
$$

Note that $\operatorname{reg}(E) \geqslant \operatorname{reg}(E / Y E)$ [T, Lemma 2.1]. By induction on $s$ we may assume that for $n \geqslant c-1$,

$$
\operatorname{reg}\left([E / Y E]_{n}\right)=(n-c+1) d+\operatorname{reg}\left([E / Y E]_{c-1}\right) .
$$

Moreover, if $n \geqslant c, n-1 \geqslant \operatorname{reg}(E)+1$. Then $\left[0_{E}: Y\right]_{n-1}=0$ by [T, Proposition 2.2]. In this case we have $K_{n-1}=E_{n-1}$. We distinguish three cases:

(1) If $\left.\operatorname{reg}\left(K_{c-2}\right)(-d)\right)>\operatorname{reg}\left([E / Y E]_{c-1}\right)+1$, using Lemma 3.6 we get $\operatorname{reg}\left(E_{c-1}\right)=\operatorname{reg}\left(K_{c-2}(-d)\right)$. From this it follows that

$$
\begin{aligned}
\operatorname{reg}\left(E_{c-1}(-d)\right) & =\operatorname{reg}\left(K_{c-2}(-d)\right)+d>\operatorname{reg}\left([E / Y E]_{c-1}\right)+d+1 \\
& =\operatorname{reg}\left([E / Y E]_{c}\right)+1 .
\end{aligned}
$$


By Lemma 3.6 we get $\operatorname{reg}\left(E_{c}\right)=\operatorname{reg}\left(E_{c-1}(-d)\right)=d+\operatorname{reg}\left(E_{c-1}\right)$. Using the same argument, we will be led to the formula $\operatorname{reg}\left(E_{n}\right)=(n-c+1) d+\operatorname{reg}\left(E_{c-1}\right)$ for $n \geqslant c-1$.

(2) If $\operatorname{reg}\left(K_{c-2}(-d)\right)<\operatorname{reg}\left([E / Y E]_{c-1}\right)+1$, using Lemma 3.6 we get $\operatorname{reg}\left(E_{c-1}\right)=\operatorname{reg}\left([E / Y E]_{c-1}\right)$. Therefore,

$$
\operatorname{reg}\left(E_{c-1}(-d)\right)=\operatorname{reg}\left([E / Y E]_{c-1}\right)+d=\operatorname{reg}\left([E / Y E]_{c}\right) .
$$

By Lemma 3.6 we get

$$
\operatorname{reg}\left(E_{c}\right)=\operatorname{reg}\left([E / Y E]_{c}\right)=d+\operatorname{reg}\left(E_{c-1}\right) .
$$

Using Lemma 3.6 again we will be led to the formula $\operatorname{reg}\left(E_{n}\right)=(n-c+1) d+$ $\operatorname{reg}\left(E_{c-1}\right)$ for $n \geqslant c-1$.

(3) If $\operatorname{reg}\left(K_{c-2}(-d)\right)=\operatorname{reg}\left([E / Y E]_{c-1}\right)+1$, then $\operatorname{reg}\left(E_{c-1}\right) \leqslant \operatorname{reg}\left(K_{c-2}(-d)\right)$. As we have seen in (1), we may assume that $\operatorname{reg}\left(E_{c-1}\right)<\operatorname{reg}\left(K_{c-2}(-d)\right)$. It follows that

$$
\operatorname{reg}\left(E_{c-1}(-d)\right)<d+\operatorname{reg}\left([E / Y E]_{c-1}\right)+1=\operatorname{reg}\left([E / Y E]_{c}\right)+1 .
$$

Following (2) we will obtain $\operatorname{reg}\left(E_{n}\right)=(n-c) d+\operatorname{reg}\left(E_{c}\right)$ for $n \geqslant c$.

COROLLARY 3.8. Let I be an ideal generated by a d-sequence of $s$ forms of the same degree $d$. For $n \geqslant s+1, \operatorname{reg}\left(I^{n}\right)=(n-s-1) d+\operatorname{reg}\left(I^{s+1}\right)$.

\section{Regularity of Saturations of Ideals}

In this section we will study the regularity of the saturation $\widetilde{I}^{n}$ of $I^{n}$.

PROPOSITION 4.1. Let I be an arbitrary homogeneous ideal. There is a number e such that $\operatorname{reg}\left(\widetilde{I}^{n}\right) \leqslant n d(I)+e$, for all $n \geqslant 1$.

Proof. We have

$$
H_{\mathfrak{m}}^{i}\left(\widetilde{I^{n}}\right) \simeq\left\{\begin{array}{l}
0, \quad i=0,1, \\
H_{\mathfrak{m}}^{i}\left(I^{n}\right), \quad i \geqslant 2 .
\end{array}\right.
$$

Hence the conclusion follows from Theorem 2.4.

Now we will present examples which show that $\operatorname{reg}\left(\widetilde{I}^{n}\right)$ is not a linear polynomial for $n \gg 0$. The ideal $I$ will be the ideal of certain 'fat' points.

EXAMPLE 4.2. Let $p_{1}, \ldots, p_{s}$ be distinct points on a rational normal curve in $\mathbb{P}^{r}$, $s \geqslant 2$. Let $\wp_{1}, \ldots, \wp_{s}$ denote their defining prime ideals in $A=k\left[X_{0}, \ldots, X_{r}\right]$, where $k$ is an arbitrary algebraically closed field, and $I=\wp_{1} \cap \cdots \cap \wp_{s}$. Then $\widetilde{I}^{n}=\wp_{1}^{n} \cap \cdots \cap \wp_{s}^{n}$. 
By [CTV, Proposition 7] we know that

$$
\operatorname{reg}\left(A / \widetilde{I}^{n}\right)=\max \left\{2 n-1,\left[\frac{n s+r-2}{r}\right]\right\} .
$$

Note that $\operatorname{reg}\left(\widetilde{I^{n}}\right)=\operatorname{reg}\left(A / \widetilde{I^{n}}\right)+1$. If $s \geqslant 2 r$, then

$$
\operatorname{reg}\left(\widetilde{I^{n}}\right)=\left[\frac{n s+2 r-2}{r}\right] \text {. }
$$

In this case, if $s$ is not divided by $r, \operatorname{reg}\left(\widetilde{I^{n}}\right)$ differs from a linear function by a periodic function whose values depend on the residue of $s$ modulo $r$.

A more precise result can be obtained in the following situation

THEOREM 4.3. Let I be a homogeneous ideal. Assume that the graded algebra $\oplus_{n \geqslant 0} \widetilde{I}^{n} t^{n}$ is finitely generated. Then there exists a positive integer $r$ and linear polynomials $f_{i}(n)=n d_{i}+e_{i}$ for $0 \leqslant i \leqslant r-1$ such that $\operatorname{reg}\left(\widetilde{I^{n}}\right)=f_{\sigma(n)}(n)$ for $n \gg 0$, where $\sigma(n) \equiv n \bmod r$.

Proof. Since $\tilde{R}=\oplus \tilde{I}^{n} t^{n}$ is finitely generated, it may be written as a factor ring of a bigraded polynomial ring $S=k\left[X_{1}, \ldots, X_{r}, Y_{1}, \ldots, Y_{s}\right]$ where $\operatorname{deg} X_{i}=$ $(1,0)$ for $i=1, \ldots, r$, and $\operatorname{deg} Y_{j}=\left(d_{j}, t_{j}\right)$ for $j=1, \ldots, s$. The arguments of Lemma 3.3 apply as well to $\tilde{R}$. So we conclude that

$$
\operatorname{reg}_{i}\left(\tilde{I^{n}}\right)=\max \left\{a \mid \operatorname{Tor}_{i}^{S}(S / N, \tilde{R})_{(a, n)} \neq 0\right\}-i .
$$

Thus the conclusion follows if we prove the following analogue of Theorem 3.4: Suppose that $E$ is a finitely generated bigraded module over $T=k\left[Y_{1}, \ldots, Y_{s}\right]$ where $\operatorname{deg} Y_{j}=\left(d_{j}, t_{j}\right)$ for $j=1, \ldots, s$. Then there exists an integer $k_{0}$ and linear functions $\ell_{i}, i=0, \ldots, k_{0}$, such that for all $n \gg 0$ one has that $\rho_{E}(n)=\ell_{i}(n)$ if $n \equiv i \bmod k_{0}$.

Consider the $\mathbb{N}$-grading $T_{b}=\oplus_{a} T_{(a, b)}$. Then there exists an integer $k_{0}$ such that the $k_{0}$ th Veronese subring $T^{\left(k_{0}\right)}=\oplus_{i \geqslant 0} T_{i k_{0}}$ of $T$ is standard graded in degree 1 (after normalizing the grading). Note that $E$ considered as an $T^{\left(k_{0}\right)}$-module decomposes as $E=\oplus_{i=0}^{k_{0}-1} T_{i} E$. Therefore we may apply 3.4, and see that the functions $\rho_{T_{i} E}(n)$ of the $T^{\left(k_{0}\right)}$-modules $T_{i} E$ are linear for $n \gg 0$.

Now let $n$ be arbitrary. Then $n=m k_{o}+i$ with $0 \leqslant i \leqslant k_{0}-1$, and $\rho_{E}^{T}(n)=$ $\rho_{T_{i} E}^{T^{\left(k_{0}\right)}}(m)$. Hence the conclusion follows.

The following example shows that in general $\operatorname{reg}\left(\widetilde{I}^{n}\right)$ is not a linear polynomial with periodic coefficients.

EXAMPLE 4.4. For any $p>0$ such that $p$ is congruent to $2 \bmod 3$, there exists a field $k$ of characteristic $p$ and an ideal $I \subset k[x, y, z]$ such that the regularity of the 
saturated powers $\widetilde{I^{n}}$ is not (eventually) periodic. In fact, $\operatorname{reg}\left(\widetilde{I^{5 n+1}}\right)=29 n+7$ if $n$ is not a power of $p$ and $\operatorname{reg}\left(\widetilde{I^{5 n+1}}\right)=29 n+8$ if $n$ is a power of $p$.

In [CS, Sect. 6] the first author and Srinivas construct a counterexample to Zariski's Riemann-Roch problem in char $p>0$. There one can find a non singular projective curve $C$ of genus 2 over a field $k$ of characteristic $p \neq 0$ as above with points $\eta, q \in C$ such that

$$
h^{1}\left(\mathcal{O}_{C}(n(\eta-q)+q)\right)= \begin{cases}0 & \text { if } n \text { is not a power of } p, \\ 1 & \text { if } n \text { is a power of } p .\end{cases}
$$

We will use this curve to construct our example.

Set $D=6 q-\eta$. Then $D$ is a divisor on $C$ such that $\operatorname{deg}(D)=5 \geqslant 2 g+1$, where $g=2$ is the genus of $C$. Thus $D$ is very ample [H2, Coro. IV.3.2] and $h^{0}\left(\mathcal{O}_{C}(D)\right)=\operatorname{deg}(D)+1-g=4$ [H2, Example IV.1.3.4 and Thm. IV.1.3]. Hence $H^{0}\left(C, \mathcal{O}_{C}(D)\right)$ gives an embedding of $C$ as a curve of degree 5 in $\mathbb{P}^{3}$. We can project $\mathrm{C}$ onto a degree 5 plane curve $\gamma$ with only nodes as singularities from a point in $\mathbb{P}^{3}$ not on $C$ [H2, Thm IV.3.10]. The arithmetic genus of $\gamma$ is $p_{a}(\gamma)=2+n$ where $n$ is the number of nodes of $\gamma$ [H2, Exercise IV.1.8]. Since $d=\operatorname{deg}(\gamma)=5$, $p_{a}(\gamma)=\frac{1}{2}(d-1)(d-2)=6[\mathrm{H} 2$, Exercise I.7.2]. Thus $\gamma$ has $n=4$ nodes.

Let these singular points be $q_{1}, \ldots, q_{4}$. Let $\pi_{1}: S_{1} \rightarrow \mathbb{P}^{2}$ be the blow up of these 4 points. Let $F_{i}$ be the exceptional curves that map respectively to $q_{i}$. Let $\gamma_{1}$ be the strict transform of $\gamma$. Then $\gamma_{1} \cong C$ since it is nonsingular. Let $H_{1}=\pi_{1}^{-1}\left(H^{\prime}\right)$ where $H^{\prime}$ is a hyperplane on $\mathbb{P}^{2}$. Since the singular points are nodes

$$
\pi^{-1}(\gamma)=\gamma_{1}+2 F_{1}+\cdots+2 F_{4}, \quad \text { and } \quad F_{i} \cdot \gamma_{1}=q_{i 1}+q_{i 2},
$$

for (distinct) points $q_{i j}$ on $\gamma_{1}, 1 \leqslant i \leqslant 4, j=1,2$. The divisor

$$
5 H_{1} \cdot \gamma_{1}-2 q_{11}-\cdots-2 q_{42}-\eta+5 q
$$

has degree 13 since $\left(H_{1} \cdot \gamma_{1}\right)=\left(H^{\prime} \cdot \gamma\right)=5$. Thus it is very ample [H2, Corollary IV.3.2], and there are points $p_{1}, \ldots, p_{13} \in \gamma_{1}$ such that

$$
5 H_{1} \cdot \gamma_{1}-2 q_{11}-\cdots-2 q_{42}-\eta+5 q \sim p_{1}+\cdots+p_{13},
$$

where $\sim$ denotes linear equivalence.

Let $\pi_{2}: S_{2} \rightarrow S_{1}$ be the blowup of the points $p_{1}, \ldots, p_{13}$, with respective exceptional curves $E_{i}$ mapping to $p_{i}$. Let $\bar{\gamma} \cong C$ be the strict transform of $\gamma_{1}, \bar{F}_{i}$ be the strict transform of $F_{i}$ for $1 \leqslant i \leqslant 4$. Let $\pi: S_{2} \rightarrow \mathbb{P}^{2}$ be the composed map. Let $\bar{H}=\pi^{-1}\left(H^{\prime}\right)$. Then

$$
\begin{aligned}
& 5 \bar{H} \sim \pi^{-1}(\gamma)=\bar{\gamma}+E_{1}+\cdots+E_{13}+2 \bar{F}_{1}+\cdots+2 \bar{F}_{4}, \\
& \bar{\gamma} \cdot \bar{\gamma} \sim\left(5 \bar{H}-E_{1}-\cdots-E_{13}-2 \bar{F}_{1}-\cdots-2 \bar{F}_{4}\right) \cdot \bar{\gamma} \sim \eta-5 q .
\end{aligned}
$$


By our construction, $\bar{H} \cdot \bar{\gamma} \sim D=6 q-\eta$. Thus

$$
(5 \bar{\gamma}+4 \bar{H}) \cdot \bar{\gamma} \sim \eta-q, \quad(\bar{\gamma}+\bar{H}) \cdot \bar{\gamma} \sim q .
$$

Set $A=5 \bar{\gamma}+4 \bar{H}, B=\bar{\gamma}+\bar{H}$. Observe that $\left(\bar{\gamma}^{2}\right)=-4$ and $(\bar{\gamma} \cdot \bar{H})=5$. $H^{1}\left(S_{2}, \mathcal{O}_{S_{2}}(m \bar{H})\right)=0$ for all $m \geqslant 0$ and $H^{1}\left(\bar{\gamma}, \mathcal{O}_{\bar{\gamma}}(m \bar{H}+n \bar{\gamma})\right)=0$ if $5 m-4 n \geqslant$ 3 since $((m \bar{H}+n \bar{\gamma}) \cdot \bar{\gamma})=5 m-4 n$ and a divisor on a curve of genus $g$ is nonspecial if its degree is $>2 g-2$ [H2, Example IV.1.3.4]. Consideration of the cohomology of

$$
\begin{aligned}
0 & \rightarrow \mathcal{O}_{S_{2}}(m \bar{H}+(n-1) \bar{\gamma}) \rightarrow \mathcal{O}_{S_{2}}(m \bar{H}+n \bar{\gamma}) \\
& \rightarrow \mathcal{O}_{\bar{\gamma}}(m \bar{H}+n \bar{\gamma}) \rightarrow 0
\end{aligned}
$$

and induction imply $H^{1}\left(S_{2}, \mathcal{O}_{S_{2}}(m \bar{H}+n \bar{\gamma})\right)=0$ if $5 m-4 n \geqslant 3$. The relations $H^{2}\left(S_{2}, \mathcal{O}_{S_{2}}(m \bar{H})\right)=0$ for all $m \geqslant 0$ and $H^{2}\left(\bar{\gamma}, \mathcal{O}_{\bar{\gamma}}(m \bar{H}+n \bar{\gamma})\right)=0$ for all $m, n$ imply $H^{2}\left(S_{2}, \mathcal{O}_{S_{2}}(m \bar{H}+n \bar{\gamma})\right)=0$ for all $m, n>0$.

For all $n \geqslant 0$ we have

$$
0 \rightarrow \mathcal{O}_{S_{2}}(n A+\bar{H}) \rightarrow \mathcal{O}_{S_{2}}(n A+B) \rightarrow \mathcal{O}_{\bar{\gamma}}(n A+B) \rightarrow 0 .
$$

By the above, $H^{1}\left(S_{2}, \mathcal{O}_{S_{2}}(n A+\bar{H})\right)=H^{2}\left(S_{2}, \mathcal{O}_{S_{2}}(n A+\bar{H})\right)=0$ for all $n \geqslant 0$. From $(*)$ we see that

$$
\begin{aligned}
h^{1}\left(\mathcal{O}_{S_{2}}(n A+B)\right) & =h^{1}\left(\mathcal{O}_{C}(n(\eta-q)+q)\right) \\
& = \begin{cases}0 & \text { if } n \text { is not a power of } p, \\
1 & \text { if } n \text { is a power of } p .\end{cases}
\end{aligned}
$$

By $\left({ }^{*}\right), H^{1}\left(S_{2}, \mathcal{O}_{S_{2}}(4 n \bar{H}+(5 n-1) \bar{\gamma})\right)=0$ for all $n>0$. Then by the RiemannRoch Theorem on $\bar{\gamma}$ and $(*)$,

$$
h^{1}\left(\mathcal{O}_{S_{2}}(4 n \bar{H}+5 n \bar{\gamma})\right)=h^{1}\left(\mathcal{O}_{\bar{\gamma}}(4 n \bar{H}+5 n \bar{\gamma})\right)=1,
$$

for $n>0$ since $((4 n \bar{H}+5 n \bar{\gamma}) \cdot \bar{\gamma})=0$ and by Riemann-Roch. $((4 n \bar{H}+(5 n+1) \bar{\gamma})$. $\bar{\gamma})=-4$. Thus $h^{0}\left(\mathcal{O}_{\bar{\gamma}}(4 n \bar{H}+(5 n+1) \bar{\gamma})\right)=0$ and $h^{1}\left(\mathcal{O}_{\bar{\gamma}}(4 n \bar{H}+(5 n+1) \bar{\gamma})\right)=5$ by Riemann-Roch. By (*) we have $h^{1}\left(\mathcal{O}_{S_{2}}(4 n \bar{H}+(5 n+1) \bar{\gamma})=4\right.$.

The formulas $\left((n A+B+m \bar{H}) \cdot E_{i}\right)>0$ and $\left((n A+B+m \bar{H}) \cdot \bar{F}_{i}\right)>0$ for all $m, n \geqslant 0$ imply that $R^{i} \pi_{*} \mathcal{O}_{S_{2}}(n A+B+m \bar{H})=0$ for $m, n \geqslant 0$, and $H^{1}\left(S_{2}, \mathcal{O}_{S_{2}}(n A+B+m \bar{H})\right)=H^{1}\left(S, \pi_{*} \mathcal{O}_{S_{2}}(n A+B+m \bar{H})\right)$. The relation

$$
\begin{aligned}
& n A+B+m \bar{H} \sim(29 n+6+m) \bar{H}- \\
& \quad-(5 n+1)\left(E_{1}+\cdots+E_{13}+2 \bar{F}_{1}+\cdots+2 \bar{F}_{4}\right)
\end{aligned}
$$


implies

$$
\begin{aligned}
& \pi_{*} \mathcal{O}(n A+B+m \bar{H})
\end{aligned}
$$

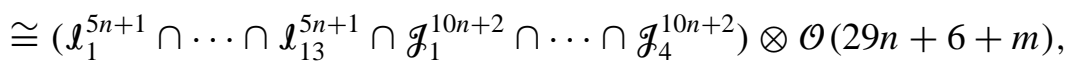

where $\ell_{i}$ are the ideal sheaves of the points $p_{i}$ and $g_{j}$ are the ideal sheaves of the points $q_{j}$ in $\mathbb{P}^{2}$.

Let $\wp_{1}, \ldots, \wp_{13}$ and $\wp_{14}, \ldots, \wp_{17}$ be the homogeneous primes in $k[x, y, z]$ which sheafify to $\ell_{1}, \ldots, \ell_{13}$ and $\mathscr{g}_{1}, \ldots, g_{4}$, respectively. Set

$$
I=\wp_{1} \cap \cdots \cap \wp_{13} \cap \wp_{14}^{2} \cap \cdots \cap \wp_{17}^{2} .
$$

Let $\mathfrak{m}=(x, y, z)$. Then

$$
\begin{aligned}
& H_{\mathfrak{m}}^{0}\left(\widetilde{I^{n}}\right)=H_{\mathfrak{m}}^{1}\left(\widetilde{I^{n}}\right)=0, \quad H_{\mathfrak{m}}^{2}\left(\widetilde{I^{n}}\right)=\oplus_{a \in \mathbb{Z}} H^{1}\left(\mathbb{P}^{2}, \ell^{n}(a)\right), \\
& H_{\mathfrak{m}}^{3}\left(\widetilde{I^{n}}\right)=\oplus_{a \in \mathbb{Z}} H^{2}\left(\mathbb{P}^{2}, l^{n}(a)\right),
\end{aligned}
$$

where $\ell$ is the ideal sheaf of $I$. Putting everything together, we obtain

$$
\begin{aligned}
& \operatorname{dim}_{k} H_{\mathfrak{m}}^{2}\left(\widetilde{I^{5 n+1}}\right)_{(s-2)}= \begin{cases}0 & \text { if } s>29 n+8, \\
0 & \text { if } s=29 n+8 \text { and } n \text { is not a power of } p . \\
1 & \text { if } s=29 n+8 \text { and } n \text { is a power of } p, \\
4 & \text { if } s=29 n+7 .\end{cases} \\
& H_{\mathfrak{m}}^{3} \widetilde{\left(I^{5(n+1)}\right)_{(s-3)}=0} \text { if } s \geqslant 29 n+7 .
\end{aligned}
$$

By Theorem 4.3 we know that $\oplus_{n \geqslant 0} \widetilde{I}^{n}$ is not a finitely generated $k$-algebra. We can verify this directly.

If $\oplus_{n \geqslant 0} \widetilde{I}^{n}$ were finitely generated, there would be a surjection of a bigraded polynomial ring onto $\oplus_{n \geqslant 0} \widetilde{I^{n}}$. Then the subalgebra $R=\oplus_{n \geqslant 0}\left(\widetilde{I^{5 n}}\right)_{29 n}$ would be finitely generated. We will show that $R$ is not finitely generated

$$
R \cong \oplus_{n \geqslant 0} H^{0}\left(S_{2}, \mathcal{O}_{S_{2}}(n A)\right) .
$$

From (*), and our calculation $H^{1}\left(S_{2}, \mathcal{O}_{S_{2}}(m \bar{H}+n \bar{\gamma})\right)=0$ if $5 m-4 n \geqslant 3$, we see that we have surjections

$$
H^{0}\left(S_{2}, \mathcal{O}_{S_{2}}(n A)\right) \rightarrow H^{0}\left(\bar{\gamma}, \mathcal{O}_{\bar{\gamma}}(n A)\right) \cong H^{0}\left(\bar{\gamma}, \mathcal{O}_{\bar{\gamma}}(n(\eta-q))\right)=0,
$$

since $\eta-q$ must have infinite order in the Jacobian of $\bar{\gamma}$, and

$$
H^{0}\left(S_{2}, \mathcal{O}_{S_{2}}(n A-\bar{\gamma})\right) \rightarrow H^{0}\left(\bar{\gamma}, \mathcal{O}_{\bar{\gamma}}(n A-\bar{\gamma})\right) \neq 0,
$$


since $(\bar{\gamma} \cdot(n A-\bar{\gamma}))=-(\bar{\gamma} \cdot \bar{\gamma})=4 \geqslant 2 g$ and by [H2, Cor. IV.3.2]. Thus the fixed locus (counting multiplicity) of the complete linear system $|n A|$ is $\bar{\gamma}$ for all $n>0$. Since this multiplicity is nonzero and bounded for all $n>0, R$ is not finitely generated (c.f. [[Z], Part I, Sect. 2]).

The following example shows interesting asymptotic behaviour for an ideal in the coordinate ring of an abelian surface. In this example, $\lim r e g\left(\widetilde{I^{n}}\right) / n$ is an irrational number. The construction is based on an example in $[\mathrm{Cu}]$.

EXAMPLE 4.4. Let $k$ be a an algebraically closed field of arbitrary characteristic. Let $C$ be an elliptic curve over $k$ and let $S=C \times C$. Let $\Delta \subset S$ be the diagonal, $P \in S$ a closed point and $A=\pi_{1}^{-1}(p), B=\pi_{2}^{-1}(P)$, where $\pi_{i}: S \rightarrow C, i=1,2$ are the projections. Let NS $(S)$ be the Neron-Severi group of $S$ and $\overline{\mathrm{NE}}(S)$ be the closure in the metric topology on $\mathrm{NS}(S) \otimes_{\mathbf{Z}} \mathbf{R}$ of the cone generated by the curves on $S$. Let $\mathcal{V} \subset \mathrm{NS}(S) \otimes_{\mathbf{Z}} \mathbf{R}$ be the real vector space with basis $\{A, B, \Delta\}$. Observe that $\left(\Delta^{2}\right)=\left(A^{2}\right)=\left(B^{2}\right)=0,(A \cdot B)=(A \cdot \Delta)=(B \cdot \Delta)=1$. Let

$$
\begin{aligned}
U & =\left\{(x, y, z) \mid(x A+y B+z \Delta)^{2}>0\right\} \\
& =\{(x, y, z) \mid(x y+x z+y z)>0\} .
\end{aligned}
$$

$U$ consists of two disjoint, connected cones. Let $G$ be the connected component containing $L=A+B+\Delta$. By the index Theorem $(E \cdot L)>0$ for any rational $E \in G$. Hence the effective classes in $\mathcal{V}$ are contained in the closure $\bar{G}$ of $G$. If $E$ is a rational class in $G$, then $E$ is ample by the Riemann-Roch Theorem, and the fact that any effective divisor on an abelian surface with a positive intersection number is ample. Hence $\bar{G}=\mathcal{V} \cap \overline{\mathrm{NE}}(S)$. Let $H=3 A+6 B+9 \Delta, D=A+B+\Delta$

$$
(s H-D)^{2}=198 s^{2}-72 s+6=0
$$

has the roots

$$
s_{1}=\frac{1}{33}(6-\sqrt{3}), \quad s_{2}=\frac{1}{33}(6+\sqrt{3}) .
$$

If $s>s_{2}$ then $s H-D$ is in the ample cone. If $s_{1}<s<s_{2}$ then $s H-D$ is not in the effective cone, and $D-s H$ is not in the effective cone.

By Mumford's Vanishing Theorem (Sect. 16 of [Mu]), if $m$ and $r$ are nonnegative integers

$$
H^{1}\left(S, \mathcal{O}_{S}(m H-r D)\right)=0 \quad \text { if } m>r s_{2}
$$

and

$$
H^{2}\left(S, \mathcal{O}_{S}(m H-r D)\right)=0 \quad \text { if } m>r s_{2} .
$$

Suppose that $m, r$ are nonnegative integers such that $s_{1} r<m<s_{2} r$. Then

$$
H^{0}\left(S, \mathcal{O}_{S}(m H-r D)\right)=0
$$


and

$$
H^{2}\left(S, \mathcal{O}_{S}(m H-r D)\right)=H^{0}\left(S, \mathcal{O}_{S}(r D-m H)\right)=0 .
$$

By the Riemann-Roch Theorem of Section 16 [Mu]

$$
\chi(m H-r D)=\frac{(m H-r D)^{2}}{2} .
$$

Thus if $s_{1} r<m<s_{2} r$ we have

$$
h^{1}(m H-r D)=-\frac{(m H-r D)^{2}}{2}>0 .
$$

$H$ is very ample on $S$ by the Lefschetz Theorem (Section 17 of [Mu]). Set $R=$ $\oplus_{n \geqslant 0} H^{0}\left(S, \mathcal{O}_{S}(n H)\right)$, with graded maximal ideal $m$. Let $I_{1}$ be the homogeneous ideal of $A, I_{2}$ be the homogeneous ideal of $B, I_{3}$ the homogeneous ideal of $\Delta$. Let $I=I_{1} \cap I_{2} \cap I_{3}$. Let $\ell$ be the sheafification of $I$. Since $H_{m}^{2}\left(\widetilde{I}^{r}\right)_{n-2} \cong H^{1}\left(S, \mathcal{O}_{S}(n-\right.$ 2) $H-r D)$ ) and $\left.H_{m}^{3}\left(\widetilde{I}^{r}\right)_{n-3} \cong H^{2}\left(S, \mathcal{O}_{S}(n-3) H-r D\right)\right)$, we have that the 'regularity' of $\widetilde{I}^{r}$ is $\left[s_{2} r\right]+2=[(r / 33)(6+\sqrt{3})]+2$.

The ring $\oplus_{n \geqslant 0} \widetilde{I^{n}}$ of Example 4.4 is not finitely generated. This follows since

$$
\left(\widetilde{I^{r}}\right)_{m}=H^{0}\left(S, \mathcal{O}_{S}(m H-r D)\right)=\left\{\begin{array}{l}
0 \text { if } m<s_{2} r, \\
\neq 0 \text { if } m>s_{2} r .
\end{array}\right.
$$

\section{Acknowledgement}

The third author would like to thank the Max-Planck-Institut für Mathematik, Bonn, for financial support and hospitality during the summer of 1997 when this paper was worked out. The authors are grateful to K. Chandler, K. Smith and I. Swanson for sending them the preprints $[\mathrm{C}]$ and $[\mathrm{SS}]$ and to G. Valla for stimulating discussions. Furthermore, we would like to thank A. Conca who informed us that Vijay Kodiyalam $[\mathrm{K}]$ has independently obtained similar results.

\section{References}

[ABW] Akin, K., Buchsbaum, D. A. and Weyman, J.: Resolutions of determinantal ideals: The submaximal minors, Advances in Math. 39 (1981), 1-30.

[BM] Bayer, D. and Mumford, D.: What can be computed in algebraic geometry? In: D. Eisenbud and L. Robbiano (eds), Computational Algebraic Geometry and Commutative Algebra, Proceedings, Cortona 1991, Cambridge University Press, 1993, pp. 1-48.

[B] Bertram, A.: An application of a log version of the Kodaira vanishing theorem to embedded projective varieties, Preprint.

[BEL] Bertram, A., Ein, L. and Lazarsfeld, R.: Vanishing theorems, a theorem of Severi, and the equations defining projective varieties, J. Amer. Math. Soc. 4 (1991), 587-602.

[BH] Bruns, W. and Herzog, J.: Cohen-Macaulay Rings, Cambridge Univ. Press, 1993. 
[CTV] Catalisano, M., Trung, N. V. and Valla, G.: A sharp bound for the regularity index of fat points in general position, Proc. Amer. Math. Soc. 118 (1993), 717-724.

[Cu] Cutkosky, S. D.: Zariski decomposition of divisors on algebraic varieties, Duke Math. J, 53 (1986), 149-156.

[CS] Cutkosky, S. D. and Srinivas, V.: On a problem of Zariski on dimensions of linear systems, Ann. of Math. 137 (1993), 531-559.

[C] Chandler, K.: Regularity of the powers of an ideal, Preprint.

[EG] Eisenbud, D. and Goto, S.: Linear free resolutions and minimal multiplicities, J. Algebra 88 (1984), 107-184.

[EH] Eisenbud, D. and Huneke, C.: Cohen-Macaulay Rees algebras and their specialisations, J. Algebra 81 (1983), 202-224.

[EK] Eliahou, S. and Kervaire, M.: Minimal resolutions of some monomial ideals, J. Algebra 129 (1990), 1-25.

[GGP] Geramita, A., Gimigliano, A. and Pitteloud, Y.: Graded Betti numbers of some embedded rational $n$-folds, Math. Ann. 301 (1995), 363-380.

[H1] Hartshorne, R.: Local Cohomology, Lecture Notes in Math. 49, Springer, New York, 1967.

[H2] Hartshorne, R.: Algebraic Geometry, Springer, 1977.

[HT] Hoa, L. T. and Trung, N. V.: On the Castelnuovo regularity and the arithmetic degree of monomial ideals, Math. Z., to appear.

[Hu1] Huneke, C.: The theory of $d$-sequences and powers of ideals, Adv. Math. 46 (1982), 249-279.

[Hu2] Huneke, C.: Uniform bounds in noetherian rings, Invent. Math. 107 (1992), 203-223.

[K] Kodiyalam, V.: Asymptotic behaviour of the Castelnuovo-Mumford regularity, Preprint 1997.

[Mu] Mumford, D.: Abelian Varieties, Oxford Univ. Press, 1974.

[S] Swanson, I.: Powers of ideals, primary decompositions, Artin-Rees lemma and regularity, Math. Ann. 307 (1997), 299-313.

[SS] Smith, K. and Swanson, I.: Linear bounds on growth of associated primes. Comm. Algebra 25(10) (1997), 3071-3079.

[T] Trung, N. V.: The Castelnuovo regularity of the Rees algebra and the associated graded ring, Trans. Amer. Math. Soc., to appear.

[W] Wahl, J.: On cohomology of the square of an ideal sheaf, J. Algebraic Geom. 6 (1997) 481-511. 